\title{
A NOTE ON KUHLMANN'S FIXED POINT THEOREMS
}

\author{
PAWEŁ GŁADKI \\ Institute of Mathematics, University of Silesia \\ ul. Bankowa 14, Katowice, Poland, 40-007 \\ and \\ Department of Computer Science, AGH University of Science and Technology \\ al. Mickiewicza 30, Kraków, Poland, 30-059 \\ E-mail: pawel.gladki@us.edu.pl
}

\begin{abstract}
We show here how recently proven fixed point theorems by Kuhlmann and Kuhlmann can be derived from classical fixed point theorems from order theory.

Key Words and Phrases: fixed point theory, order theory.

2010 Mathematics Subject Classification: 03E04, 47H10, 54H25.

\section{REFERENCES}

[1] N. Bourbaki, Sur le théorème de Zorn, Arch. Math., 2(1949), 434-437.

[2] P.M. Cohn, Universal Algebra, Harper and Row, New York, 1965.

[3] B. Knaster, Un théorème sur les fonctions d'ensembles, Ann. Soc. Polon. Math., 6(1928), 133134.

[4] F.-V. Kuhlmann, K. Kuhlmann, A common generalization of metric and ultrametric fixed point theorems, Forum Math. 27 (2015), 303-327.

[5] G. Markovsky, Chain-complete posets and directed sets with applications, Algebra Univ., 6(1976), 53-68.

[6] A. Tarski, A lattice-theoretical fixpoint theorem and its applications, Pacific J. Math., 5(1955), 285-309.

[7] M. Tasković, On an equivalent of the axiom of choice and its applications, Math. Japon., 31(1986), 979-991.

[8] E. Witt, Beweisstudien zum Satz von M. Zorn, Math. Nachr., 4(1951), 434-438.
\end{abstract}

Received: November 7, 2014; Accepted: February 5, 2015. 